\title{
Stroke caused by carotid artery dissection during ablation of atrial arrhythmia
}

\author{
Maciej Raczak ${ }^{1}$, Anetta Lasek-Bal², Andrzej Hoffmann ${ }^{1}$, Katarzyna Mizia-Stec ${ }^{1}$, Anita Czapska ${ }^{3}$, \\ Anna M. Wnuk-Wojnar ${ }^{1}$ \\ ${ }^{1} 1^{\text {st }}$ Department of Cardiology, Medical University of Silesia, Upper Silesian Medical Centre, Katowice, Poland \\ ${ }^{2}$ Stroke Unit, Department of Neurology, Medical University of Silesia, Upper Silesian Medical Centre, Katowice, Poland \\ ${ }^{3}$ Department of Diagnostic Imaging, Medical University of Silesia, Upper Silesian Medical Centre, Katowice, Poland
}

\begin{abstract}
A bstract
A 55-year-old woman was admitted to the Cardiology Department due to a poorly tolerated attack of tachyarrhythmia. The patient was enrolled in the electrophysiology study (EPS) study with radiofrequency ablation (RF). After a few applications, during transseptal puncture, the patient demonstrated a neurological syndrome in the form of progressive aphasia and weakness in the right extremities. Computed tomography angiography showed dissection of the left common carotid artery and internal carotid artery with a thrombus within their lumen. Intravenous thrombolytic therapy was initiated, which resulted in an improvement in the patient's neurological status.
\end{abstract}

Key words: dissection of carotid artery, stroke, ablation.

\section{Introduction}

Connective tissue diseases are significant risk factors for both the heart and the walls of blood vessels of different calibers. They also increase the risk of arrhythmias, pathological heart remodeling and stroke. Every therapeutic intervention, particularly those that are invasive, should be preceded by a complex assessment of patients with connective tissue pathology.

\section{Case report}

A 55-year-old woman with a history of migraine but without a confirmed diagnosis of connective tissue disease was admitted to the Cardiology Department after few incidents of supraventricular tachycardia due to a poorly tolerated attack of tachyarrhythmia. The ECG recorded atrial tachycardia with a narrow QRS complex at a frequency of 180 beats per min. Echocardiography showed an enlargement of the left atrium with no evidence of contractility disorders. As attempts of pharmacological treatment were unsuccessful, an electrical cardioversion was performed. The effect of the procedure was short-lived and the pa- tient was qualified for the electrophysiological study with atrial mapping using a three-dimensional electroanatomical system (CARTO 3).

Firstly, a ten-pole diagnostic electrode was introduced into the coronary sinus through right subclavian vein access, then a four-pole electrode was inserted into the left femoral vein and advanced into the right atrium. The left-atrial tachyarrhythmia was excluded through entrainment stimulation and then the mapping catheter was inserted into the right atrium via the right femoral vein. The focus of arrhythmia was found and radiofrequency (RF) energy was applied at a few points. A modification of the arrhythmia was achieved - a change of the P wave cycle and morphology. After locating and performing three ablations that were focused on the right atrial arrhythmia, the next episode of left atrial tachycardia was registered. A transseptal puncture and brief mapping of the left atrium were performed.

At this point the patient demonstrated a neurological syndrome in the form of progressive aphasia and weakness in the right extremities. After an emergency neurological consultation, a computed tomography (CT) of the

\section{Corresponding author:}

Maciej Raczak MD, $1^{\text {st }}$ Department of Cardiology, Medical University of Silesia, Upper Silesian Medical Centre, 45-47 Ziołowa St, 40-635 Katowice, Poland, phone: +48 507848 411, e-mail: m.raczak@tlen.pl

Received: 7.02.2014, accepted: 11.04.2014. 
head and a CT angiography of the carotid and cerebral arteries were performed and then the patient was admitted to the Department of Neurology. Upon admission, during the neurological examination the patient was sleepy and her head and eyes were rotated to the left with a complete mixed aphasia and right-sided hemiplegia. The CT scans showed no acute lesions, which allowed an intracranial hemorrhage to be excluded and suggested an ischemic type of stroke. The CT angiography showed a dissection of the left common carotid artery (CCA) and internal carotid artery (ICA) to the level of the M1 middle cerebral artery (MCA) artery with the thrombus within the lumen of the left CCA and ICA. An intracranial CT also revealed the presence of an aneurysm of the anterior communicating artery (ACA) complex, which was $5.5 \mathrm{~mm}$ $\times 5.1 \mathrm{~mm}$ in diameter (Figures 1 and 2).

After $40 \mathrm{~min}$ from the onset of the stroke, intravenous thrombolytic therapy was initiated and an improvement in the neurological status was observed.

During the second day of the patient's hospitalization on the Neurological Ward, motor aphasia was the only neurological disorder that was observed; the patient could only say single words. A follow-up CT scan did not show any focal lesions. Intravenous heparin was added to the therapy and speech disorder therapy was started. On the fourth day, correct blood flow parameters in carotid arteries were observed during a carotid ultrasonography. However, intracranial ultrasonography showed an asymmetry of velocity with hyperkinesis in the left MCA, which was almost certainly the result of recanalization. No significant deviations in the basic blood tests were observed during the patient's hospitalization. The parameters of the coagulation system and the anticardiolipin antibody titer were normal. Other laboratory tests showed elevated levels of anti-nuclear and anti-dsDNA antibodies. No significant deviations were found during the echocardiography examination. During the further

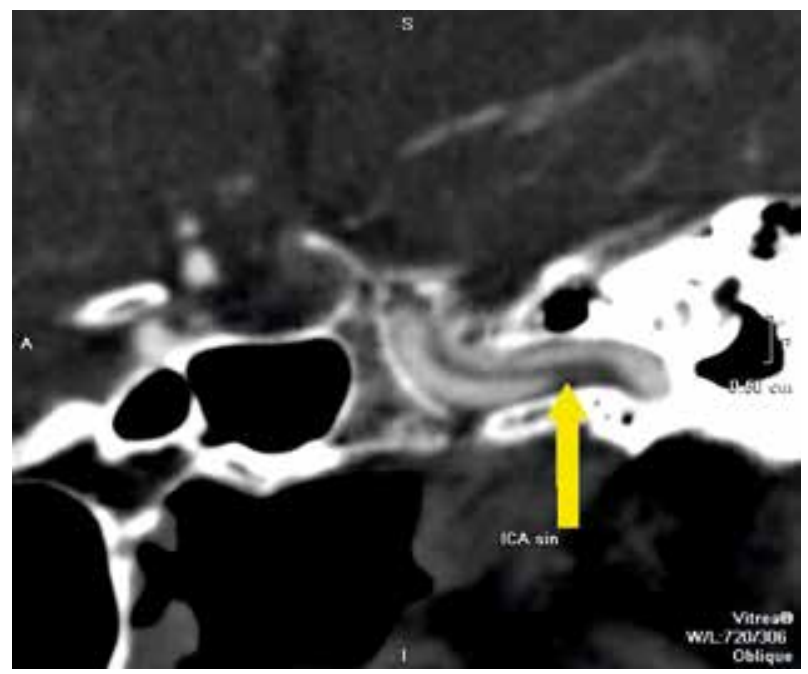

Figure 1. Dilatation of ICA sin with thrombus therapeutic process speech function was improved and on the day of discharge ( $10^{\text {th }}$ day of hospitalization), the motor aphasia still persisted, but had only a slight effect on communication with the patient. As a secondary preventative measure against brain stroke, anticoagulation therapy with rivaroxaban was introduced and further treatment of speech disorders was recommended. Blood tests, such as those to check the concentration of proteins S and C, factor VIII and other tests to check the factor $V$ Leiden mutation and prothrombin gene mutation 20210A were scheduled. Control CT angiography was performed after a month and the patient was shortlisted for endovascular embolization of an ACA aneurysm. The CT angiography of the carotid arteries revealed the correct view of the carotid arteries and the presence of an aneurysm with similar diameters as had been observed earlier (Figures 3 and 4).

\section{Discussion}

The case report concerns the spontaneous dissection of CCA and ICA during the ablation of atrial tachycardia. The dramatic neurological status of the patient - symptoms of acute cerebral ischemia - were the reason for the immediate diagnostics and subsequent thrombolytic therapy. An embolic etiology of the stroke, which could be associated with the patient's tachyarrhythmia and introduction of transseptal complex cauterization and ablation electrode to the left atrium, was assumed from the first moment that the patient was examined. The anamnesis of paroxysmal atrial fibrillation, which is an important risk factor of brain stroke, seemed to confirm this hypothesis. Moreover, the status of a patient with a cardiogenic brain stroke is usually severe, which is consistent with the clinical status of our patient. Cardiogenic, embolic material is usually large in diameter, which leads to an occlusion of the carotid or cerebral arteries and acute ischemia of extensive parts of the brain. A cardiogenic clot that is rich in fibrin is easier to treat using

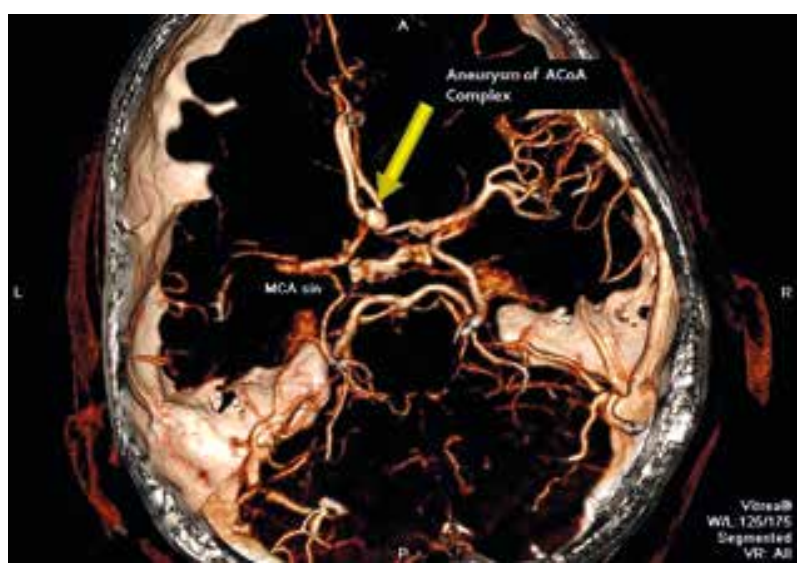

Figure 2. Aneurysm of anterior connective artery complex 


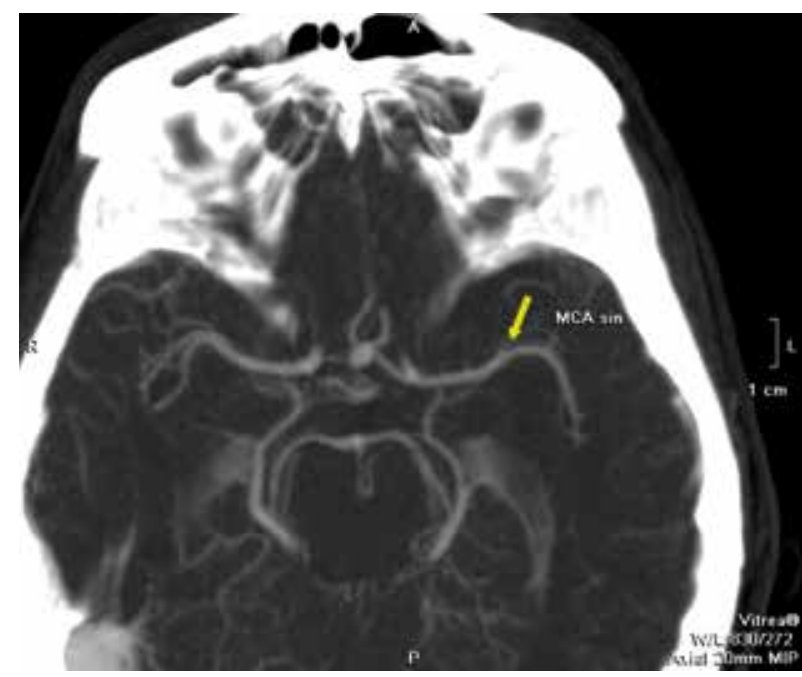

Figure 3. The MCA sin 8 weeks after dilatation

alteplase, which results in higher efficiency of artery recanalization. Therefore, the results of image examinations were surprising. A CT angiography scan revealed the dissection of the left CCA and ICA, which had a clot in their lumen. We concluded that an arterial embolism and hemodynamic insufficiency, which were the result of artery dilatation, were the most likely mechanisms that caused the patient's stroke. Application of thrombolytic therapy brought about a significant improvement of health status of the patient, despite the multiple pathomechanism of the ischemia.

Carotid and vertebral artery dissection is more frequent in comparison to that of intracranial arteries and may be spontaneous or posttraumatic [1]. It only occurs in about $2 \%$ of patients with stroke. However, it also occurs in about $25 \%$ of patients who have an acute stroke before 65 years of age. The annual prevalence of ICA dissection is 3/100 000 and 1.5/100 000 in the case of vertebral artery (VA). Although the mechanism of spontaneous artery dissection is unclear, $68 \%$ of patients had changes in the characteristics of the ultrastructural vascular walls due to connective tissue disease, regardless of its presence in the skin, articulations and other organs [2]. The localization of an ICA dissection is different from the changes that are present in atherosclerosis and are mostly situated in the middle part of an artery or in the intracranial region. A connection between the spontaneous dissection of an artery with migraine has not been established, although it is known that migraine occurs along with dissection of the artery in $49-60 \%$ of patients $[3,4]$. It is worth mentioning that migraine was also diagnosed in our patient. The most severe consequence of carotid artery dissection is acute brain ischemia (observed in $75-80 \%$ of patients) and acute retinal ischemia (5\% of patients) [5]. In the most common cases, the stroke occurs in the basin of the MCA, which suggests that there is

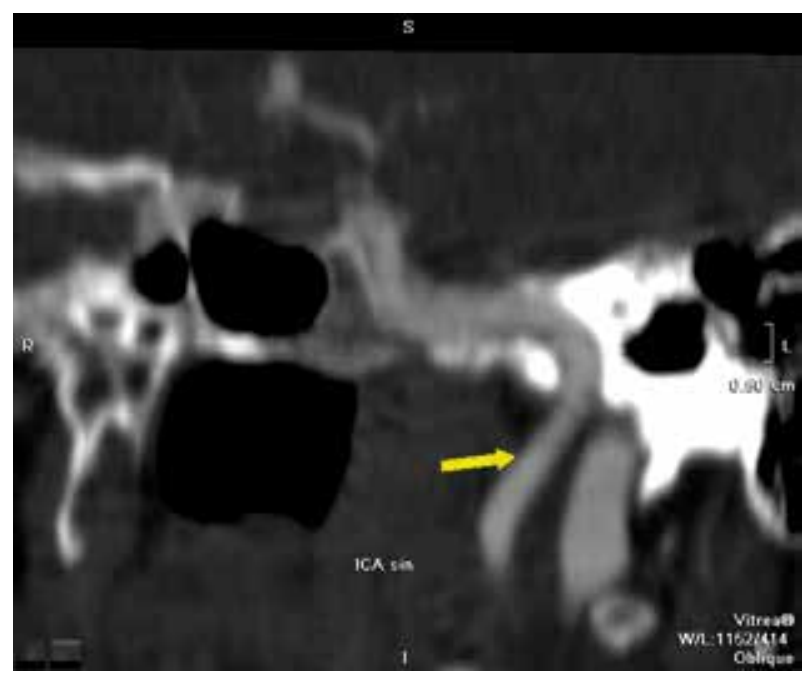

Figure 4. The ICA sin 8 weeks after dilatation

an embolic mechanism of the ischemia. The localization of ischemia in the border-zone regions of brain has also been described. It is an effect of hemodynamic insufficiency and low cerebral perfusion. In over $90 \%$ patients with artery dissection, a USG reveals disorders in cephalad arteries. Radiologic examinations show stenosis (in $50 \%$ ), occlusion (in 30-50\%) and a pseudoaneurysm (in $20 \%$ ) in these patients $[6,7]$.

Anticoagulant therapy is recommended during the acute phase of the disease and within 3 to 6 months after brain embolization. Regardless of any satisfactory efficiency, this increases the risk of a hemorrhagic stroke and the development of an intramural hematoma of the dissected artery.

Artery recanalization, which was observed in our patient, is a frequent natural progress of dissection. According to many publications, this type of progress affects as many as $70 \%$ of patients. We did not observe an ICA aneurysm (spurious aneurysm) that was connected to the dissection, which has been described in $13-49 \%$ of patients $[8,9]$.

The prognosis in patients with carotid artery dissection is relatively good, regardless of the time of the recanalization or the duration of the artery occlusion. In the case of the reocclusion of the artery, the annual index of recurrence of stroke in the region supplied by the dissected artery is $0.3 \%$. In the case of occlusion, the index is $0.7 \%$ [10]. The annual risk of recurrence of dissection of the carotid artery is less than $1 \%$ [11].

Considering the course and the results of additional examinations, it might be assumed that abnormalities of the carotid artery walls, which are associated with connective tissue disease, are the primary cause of a stroke. The hypothesis of a vascular phenotype in patients' disorders in other organs, which may relate to our patient, is also interesting [12]. We excluded any potential traumat- 
ic influence of the ablation instruments on the arteries because of the contralateral location of the pathology and the area of the operation of the interventional cardiologist. An arrhythmia or the cardiologist's intervention probably did not play any role in the acute brain stroke. Only the time of the acute dissection of the carotid artery and the cardiac intervention was concurrent.

The patient is currently undergoing oral anticoagulant therapy. She stopped the immunosuppressive therapy of connective tissue disease. She has not made a decision about endovascular intervention of the ACA complex aneurysm.

\section{References}

1. Mokri B. Traumatic and spontaneous extracranial internal carotid artery dissections. J Neurol 1990; 237: 356-61.

2. Brandt T, Hausser I, Orberk E, et al. Ultrastructural connective tissue abnormalities in patients with spontaneous cervicocerebral artery dissections. Ann Neurol 1998; 44: 281-5.

3. Pezzini A, Granella F, Grassi M, et al. History of migraine and the risk of spontaneous cervical artery dissection. Cephalalgia 2005; 25: $575-80$.

4. Tzourio C, Benslamia L, Guillon B, et al. Migraine and the risk of cervical artery dissection: a case-control study. Neurology 2002; 59: 435-7.

5. Baumgartner RW, Arnold M, Baumgartner I, et al. Carotid dissection with and without ischemic events: local symptoms and cerebral artery findings. Neurology 2001; 57: 827-32.

6. Pelkonen $\mathrm{O}$, Tikkakoski T, Leinonen S, et al. Extracranial internal carotid and vertebral artery dissections: angiographic spectrum, course and prognosis. Neuroradiology 2003; 45: 71-7.

7. Dziewas R, Sörös P, Konrad C, et al. Placing nasogastric tubes and intracranial pressure. J Neurosurg Anesthesiol 2003; 15: 55-6.

8. Touzé E, Randoux B, Méary E, et al. Aneurysmal forms of cervical artery dissection: associated factors and outcome. Stroke 2001; 32: 418-23.

9. Guillon B, Brunereau L, Biousse V, et al. Long-term follow-up of aneurysms developed during extracranial internal carotid artery dissection. Neurology 1999; 53: 117-22.

10. Kremer C, Mosso M, Georgiadis D, et al. Carotid dissection with permanent and transient occlusion or severe stenosis: long-term outcome. Neurology 2003; 60: 271-5.

11. Schievink WI, Roiter V. Epidemiology of cervical artery dissection. Front Neurol Neurosci 2005; 20: 12-5.

12. Valdueza JM, Schreiber SJ, Roehl JE, et al. Neurosonology and Neuroimaging of Stroke. Georg Thieme Verlag, Stuttgart 2008. 